\title{
Gallstone Patients with Enhanced Oxidative Stress Biomarker Superoxide Dismutase (SOD1) Plasma Levels Have Significantly Lower Number of Postoperative Analgesic Oxycodone Doses: A Prospective Study with Special Reference to Cancer Patients
}

\author{
JARI KÄRKKÄINEN ${ }^{1 *}$, IINA SAIMANEN $^{1 *}$, TUOMAS SELANDER $^{2}$, SAMULI ASPINEN $^{1}$, \\ JUKKA HARJU $^{3}$, PETRI JUVONEN ${ }^{1}$ and MATTI ESKELINEN ${ }^{1}$ \\ ${ }^{1}$ Department of Surgery and ${ }^{2}$ Science Service Center, Kuopio University Hospital, Kuopio, Finland; \\ ${ }^{3}$ Abdominal Centre, Helsinki University Hospital, Helsinki, Finland
}

\begin{abstract}
Background/Aim: Oxidative stress biomarker superoxide dismutase (SOD1) plasma levels in operated gallstone patients versus cancer patients are unknown. In addition, the number of analgesic doses during the first $24 \mathrm{~h}$ postoperatively $\left(\mathrm{NAD}_{24}\right)$ in gallstone patients operated with laparoscopic cholecystectomy (LC) or minicholecystectomy $(M C)$ is unreported. The aim of the study was to determine a correlation between the plasma SODI levels in the LC and MC patients versus cancer patients. Patients and Methods: Initially, 114 patients with symptomatic gallstone disease were randomized into LC $(n=54)$ or MC $(n=60)$ groups. The plasma levels of the SOD1 marker were measured just before, immediately after (POP1) and 6 h after the operation (POP2). Results: The median plasma SOD1 levels preoperatively and following surgery in the LC and MC patients versus cancer patients were statistically insignificant $(p=0.90, p=0.88$, $p=0.21$, respectively). The median plasma levels of SOD1 increased immediately after operation (POP1) and the postoperative elevation between the preoperative (PRE) and the POP1 values in the SOD1 marker were statistically significant ( $p=0.027)$. Then the median plasma levels of SOD1 marker decreased 6 hostoperatively (POP2) and the decrease between the POP1 and POP2 values in the SOD1 marker were
\end{abstract}

*These Authors equally contributed to this work.

Correspondence to: Matti Eskelinen, MD, Ph.D., School of Medicine, University of Eastern Finland, P.O. Box 100, FI-70029 KYS, Finland. Tel: +358 17173311, Fax: +358 17172611, GSM: +358 400969444, e-mail: matti.eskelinen@kuh.fi

Key Words: Cancer, gallstone, cholecystectomy, oxidative response, SOD1, number of analgesic doses. statistically highly significant $(p<0.001)$. There is a highly significant inverse correlation between the individual values of the $N A D_{24}$ and plasma SOD1 values postoperatively in LC and $M C$ patients $(r=-0.335, p=0.011)$. Conclusion: The plasma SOD1 levels preoperatively and following surgery in the LC and MC patients versus cancer patients were quite similar. Cholecystectomy patients with enhanced levels of SOD1 appeared to have significantly lower number of analgesic oxycodone doses during the first $24 \mathrm{~h}$ postoperatively $\left(N A D_{24}\right)$.

Gallstone disease is common among the western population and the incidence in general varies between 5-22\% (1). The treatment of symptomatic gallstone disease is mainly surgical and a complication of cholelithiasis is indication for early cholecystectomy. The classical open cholecystectomy has been replaced by mini-invasive procedures, such as minicholecystectomy (MC) and laparoscopic cholecystectomy (LC). The $\mathrm{LC}$ is the golden standard operative technique, although the MC has been shown to be as safe and effective as the LC (2-4).

"The oxidative stress concept" is a biological condition in which the generation of reactive oxygen species (ROS) is higher than the capacity of detoxifying system. ROS are mainly the result of oxygen metabolism (5). Oxidative stress is associated with several acute and chronic diseases from Alzheimer's disease to diabetes (6). A good glycemic control in diabetes has been associated with decreased oxidative stress (7), while hyperglycemia has been shown to cause increased oxidative stress (8). Yiannakopolou et al. (9) reviewed clinical trials that investigated the oxidative stress in open surgery versus laparoscopic surgery. They found four randomized clinical trials that investigated the effect of laparoscopy cholecystectomy on oxidative stress (10-13). We have earlier investigated the role of oxidative stress in open and laparoscopic surgery (14-17) and concluded that midline 
laparotomy may cause a reduction in oxidative stress biomarkers 8-OHdG (15) and GPX1 (16) plasma levels resulting in biochemical evidence of oxidative stress. However, the assessment of the oxidative stress biomarker superoxide dismutase (SOD1) plasma levels in gallstone patients versus cancer patients is unknown. The plasma levels of the SOD1 marker were measured just before, immediately after (POP1) and $6 \mathrm{~h}$ after operation (POP2). Firstly, the endpoint of this study was to determine a correlation between the plasma SOD1 levels in the LC and MC patients versus cancer patients and secondly, to determine a correlation between the plasma SOD1 levels and analgesic doses during the first $24 \mathrm{~h}$ postoperatively $\left(\mathrm{NAD}_{24}\right)$ in gallstone patients undergoing cholecystectomy.

\section{Patients and Methods}

The study was approved by the Ethics Committee of Helsinki and Uusimaa University District, Helsinki, Finland (DNRO $120 / 13 / 02 / 02 / 2010$, May 12, 2010), it was registered in the ClinicalTrials.gov database (ClinicalTrials.gov Identifier: NCT01723540, Consort diagram, Figure 1), and it was conducted in accordance with the Declaration of Helsinki. Design of the study was a prospective, randomized, multicentre clinical trial consisting of 114 patients with confirmed symptomatic cholelithiasis. Written consent was collected from participants, after receiving verbal and written information about the study. Patients were randomized to undergo either laparoscopic cholecystectomy $(n=54)$ or minicholecystectomy $(n=60)$ in two separate hospitals in Finland: Kuopio University Hospital, Kuopio $(\mathrm{n}=86)$ and Helsinki University Central Hospital, Helsinki $(n=28)$ between March 2013 and May 2015. Design of the study is presented in Figure 1. After patient enrolment, randomization was done with a sealed envelope method either to LC or MC groups. The cancer patients, recruited from our previous study, included ten patients with gastrointestinal cancer and 19 patients with gynecological cancer (18).

The surgical techniques used were standardized in both groups. The LC procedure was performed using the four-trocar technique (two $10-\mathrm{mm}$ and two 5-mm trocars) and intra-abdominal pressure was set at $12 \mathrm{mmHg}$. The ultrasonic scissors (Harmonic $\mathrm{ACE}^{\circledR}$, Ethicon Endo-Surgery, Cincinnati, OH, USA) were used both in the MC and LC procedure. The gallbladder was dissected from the liver with ultrasonic scissors. The cystic artery was sealed with ultrasonic scissor and two metal clips were inserted to the cystic duct.

The MC technique is mini-invasive open technique with very short wound; mean (SD) length of skin incision, 4.8 (1.6) cm, and the rectus muscle was not cut, but split. Skin incision length more than $7 \mathrm{~cm}$ or cutting of rectus muscle was considered as a conversion to conventional open operation. (3). Scin incisions were infiltrated with local anaesthetic $(20 \mathrm{ml}$ ropivacaine $7.5 \mathrm{mg} / \mathrm{ml})$ at the end of the operation. Both study groups were standardized regarding endotracheal anaesthesia and postoperative care including the overall pain surveyed and filed on a 11-point numeric rating scale following surgery (NRS; $0=$ no pain; $10=$ =worst pain).

For postoperative rescue analgesia, the patients were given oxycodone $3 \mathrm{mg} i . v$. if the pain at rest was NRS $3 / 10$ or higher or during cough, and/or movement the NRS was $5 / 10$ or higher. The total number of oxycodone $3 \mathrm{mg}$ doses during the first $24 \mathrm{~h}$

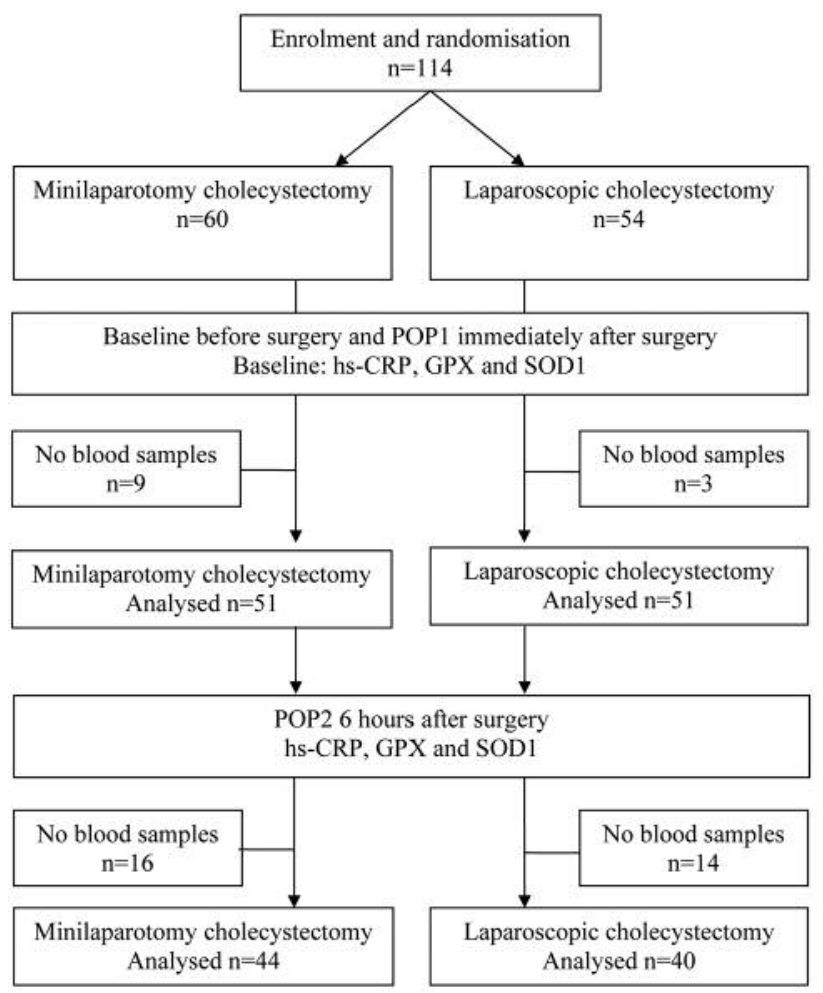

Figure 1. Design of the study as flowchart.

Table I. Baseline demographic characteristics and surgical data for the two study groups. Values are mean (standard deviation) or *number of cases. T-test and Fisher's exact test* were used.

\begin{tabular}{lccr}
\hline Variable & $\begin{array}{c}\text { Minilaparotomy } \\
\mathrm{n}=60\end{array}$ & $\begin{array}{c}\text { Laparoscopy } \\
\mathrm{n}=54\end{array}$ & $p$-Value \\
\hline Age (years) & $50.7(13.2)$ & $53.2(13.1)$ & 0.316 \\
Gender (male/female)* & $11 / 49$ & $18 / 36$ & 0.066 \\
Height (cm) & $167.4(7.6)$ & $168.9(9.9)$ & 0.355 \\
Weight (kg) & $77.3(14.4)$ & $83.0(17.4)$ & 0.057 \\
BMI (kg/m $\left.{ }^{2}\right)$ & $27.6(4.4)$ & $29.1(5.6)$ & 0.111 \\
Operative time (min) & $70.0(26.9)$ & $70.1(35.3)$ & 0.976 \\
Time in the operation & $120.4(28.5)$ & $127.1(35.8)$ & 0.287 \\
theatre (min) & & & \\
Perioperative bleed (ml) & $41(59)$ & $31(39)$ & 0.290 \\
Conversion rate (n)* & 3 & 3 & 1.000 \\
Length of the skin & $49.7(12.1)$ & $78.3(22.7)$ & $<0.001$ \\
incision(s) (mm) & & &
\end{tabular}

postoperatively $\left(\mathrm{NAD}_{24}\right)$ for cholecystectomy patients was very carefully recorded in the patient records. The study protocol was fully described in our earlier original work (19).

EDTA-blood samples were taken at the prespecified time-points and centrifuged at $1,000 \times \mathrm{g}(2,900 \mathrm{rpm})$ for $15 \mathrm{~min}$. Plasma was separated and stored frozen at $-70^{\circ} \mathrm{C}$ until analyzed. The plasma 
Table II. Changes in plasma levels of hs-CRP, GPX1 and SOD1 measured at three time points; before the operation (PRE), immediately after the operation (POP1) and $6 \mathrm{~h}$ after the operation (POP2) in minicholecystectomy (MC) and laparoscopic cholecystectomy (LC) patients versus cancer patients. Values are median (interquartile range).

\begin{tabular}{lcccc}
\hline Marker & MC & LC & Cancer & $p$-Value \\
\hline Hs-CRP (mg/l) & & & & \\
PRE & $1.45(0.49-3.1)$ & $1.70(0.56-3.65)$ & $5.5(1.0-63)$ & 0.09 \\
POP1 & $1.50(0.50-3.0)$ & $1.63(0.6-3.45)$ & $8.61(0.8-59)$ & 0.08 \\
POP2 & $3.00(1.4-5.7)$ & $2.55(0.98-4.38)$ & $209.8(101-375)$ & 0.06 \\
GPX1 (pg/ml) & $8.2(5.7-13.8)$ & $9.1(5.5-16.8)$ & $10(6.3-19)$ & 0.41 \\
PRE & $8.7(6.3-14.4)$ & $8.0(5.4-13.5)$ & $10.7(7.4-22.4)$ & 0.40 \\
POP1 & $10.4(5.1-14.6)$ & $10.2(6.6-15.6)$ & $8.1(5.2-12.3)$ & 0.61 \\
POP2 & $150(99-204)$ & $149(83-222)$ & $160(107-211)$ & 0.90 \\
SOD1 $(\mathrm{pg} / \mathrm{ml})$ & $164(118-241)$ & $195(108-260)$ & $202(136-338)$ & 0.88 \\
PRE & $130(92-168)$ & $150(116-231)$ & $112(86-196)$ & 0.21 \\
POP1 & & &
\end{tabular}

GPX1 assays were performed using sandwich-type ELISA methods from BioVendor GPX1 ELISA Kit (62100 Brno, Czech Republic, www.biovendor.com). Plasma hs-CRP was analyzed with a Cobas 6000-analyzer (Hitachi, Tokyo, Japan) using the method by Roche Diagnostics (Mannheim, Germany). The plasma SOD1 assays were performed using sandwich-type ELISA methods from BioVendor $\mathrm{Cu} / \mathrm{Zn}$ SOD ELISA Kit $(62100$ Brno, Czech Republic, www.biovendor.com). In our own analysis $(n=25)$ of the intra-assay CV\% and the inter-assay CV\% were $4.9 \%$ and $10.7 \%$, respectively.

The data were entered and analyzed with a statistical software program (IBM SPSS Statistics 24.0, IBM, Somers, IL, USA). Baseline characteristics between groups in Table I were tested by Fisher's exact test and in case of continuous data the analysis was performed by analysis of variance (ANOVA). Group differences in three time points were tested by the Mann-Whitney $U$-test in Table II and the Wilcoxon signed rank test was used in Table III. The results of the laboratory measurements are presented as median with interquartile range as distributions were right skewed. A two-sided $p$-value of less than 0.05 was considered statistically significant. The results of the individual GPX1 and SOD1 values postoperatively (POP1) for LC and MC patients are shown as jitter plots with Spearman's correlation coefficients in Figure 2. The results of the individual postoperative (POP2) SOD1 values versus number of analgesic doses during the first 24 h postoperatively $\left(\mathrm{NAD}_{24}\right)$ for cholecystectomy patients are shown as jitter plots with Spearman's correlation coefficients in Figure 3.

\section{Results}

The demographic variables and surgical data were quite similar in the study groups. There was a trend for higher mean body weight in the LC versus the MC patients $(83.0 \mathrm{~kg} v s .77 .3 \mathrm{~kg}$, $p=0.057$, Table I). However, there was no statistically significant difference in the mean body mass index values (BMI) between the $\mathrm{LC}$ and MC patients $\left(29.1 \mathrm{~kg} / \mathrm{m}^{2}\right.$ vs. $27.6 \mathrm{~kg} / \mathrm{m}^{2}, p=0.111$, Table I). Interestingly, the mean length of the skin incision was significantly longer in the LC patients than in the MC patients (78.3 mm vs. $49.7 \mathrm{~mm}, p<0.001$, Table I).
The median plasma hs-CRP levels preoperatively and following surgery in the LC and MC patients were quite similar $(p=0.42, p=0.43, p=0.63$, respectively, $p$-value between LC and MC groups, Table II). Also, the median plasma hs-CRP levels preoperatively and following surgery in the LC and MC patients versus cancer patients were statistically insignificant $(p=0.09, \quad p=0.08, p=0.06$, respectively, Table II), although there was a trend for higher postoperative plasma hs-CRP levels in cancer patients (Table II). The median plasma GPX1 levels preoperatively and following surgery in the LC and MC patients versus cancer patients were statistically insignificant $(p=0.41, p=0.40$, $p=0.61$, respectively, Table II). Also, the median plasma SOD1 levels preoperatively and following surgery in the LC and MC patients versus cancer patients were statistically insignificant ( $p=0.90, p=0.88, p=0.21$, respectively, Table II).

The median plasma level of hs-CRP increased by $7.4 \%$ immediately after surgery and this increase was statistically significant ( $p=0.021$, Table III). The median hs-CRP level then increased by $62.1 \%$ at $6 \mathrm{~h}$ postoperatively $(p<0.001$, Table III). The median plasma oxidative stress marker SOD1 levels preoperatively and following surgery in the LC and MC patients were quite similar $(p=0.891, p=0.875, p=0.207$, respectively, Table II). The median plasma level of SOD1 increased by $14.1 \%$ immediately after surgery and this increase was statistically significant ( $p=0.027$, Table III). The median SOD1 level then decreased by $21.3 \%$ at $6 \mathrm{~h}$ postoperatively $(p<0.001$, Table III).

There was a highly significant correlation between SOD1 and GPX1 values immediately after operation $(\mathrm{r}=0.638$, $p<0.001$, Figure 2). Interestingly, there was a highly significant inverse correlation between the individual values of the $\mathrm{NAD}_{24}$ and plasma SOD1 values postoperatively in LC and MC patients ( $\mathrm{r}=-0.335, p=0.011$, Figure 3$)$. 


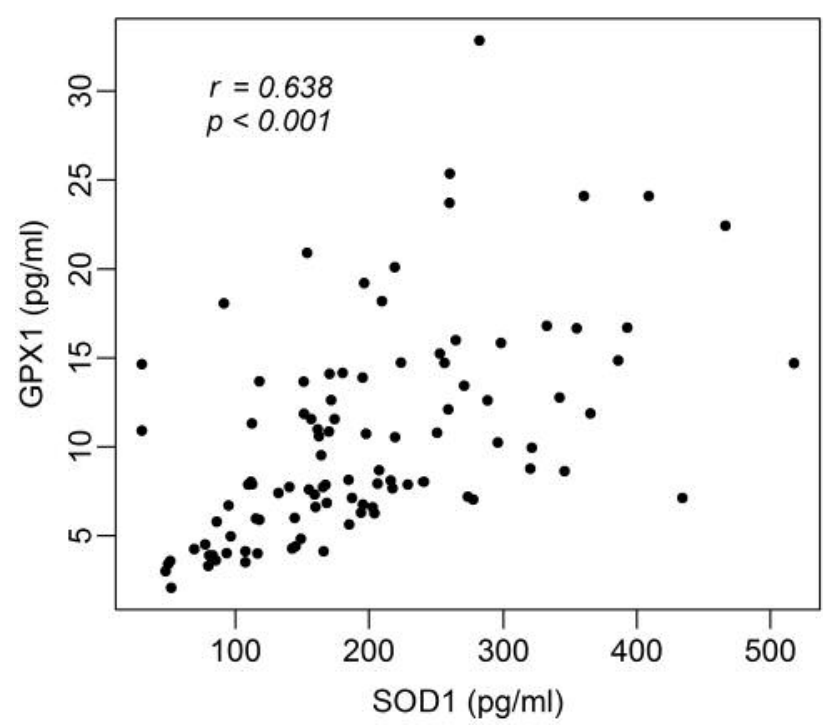

Figure 2. Scatter plots of the post-operative (POP1) plasma superoxide dismutase (SOD1) values versus glutathione peroxidase (GPX1) in cholecystectomy patients $(r=0.638, p<0.001)$.

\section{Discussion}

Many methods and markers have been used in early detection of oxidative stress and several issues need to be taken in consideration in oxidative stress research. One of the problems encountered with the oxidative stress biomarker analysis is the sample preparation. The blood sample from study patients should be standardized and quickly stabilized. The stabilization of the sample needs to be done by deep freezing after centrifugation. On this basis, in this study the EDTA-blood samples were taken at the prespecified timepoints and centrifuged at $1000 \mathrm{~g}$ for $15 \mathrm{~min}$. Plasma was separated and stored frozen at $-70^{\circ} \mathrm{C}$ until analyzed. Therefore, it is unlikely that there is a study bias from the blood sampling and/or the blood sample preparation and it is possible to compare the study group, even though, research laboratories are facing problems reproducing the data from sample to sample and from study to study (20).

Oxidative stress has an important role in the pathogenesis of toxic liver disease (21), atherogenesis (22) and is the cause of several acute and chronic conditions from oxidative stress-mediated biomolecular damage and inflammation in tumorigenesis (23) to oxidative stress during chronic alcoholism (24). Although, in the critical care patient's low plasma levels of antioxidant micronutrients have been shown, these low plasma levels are difficult to interpret since in CCU patients the multiorgan failure causes redistribution of micronutrients by cytokine mediated mechanisms (25).

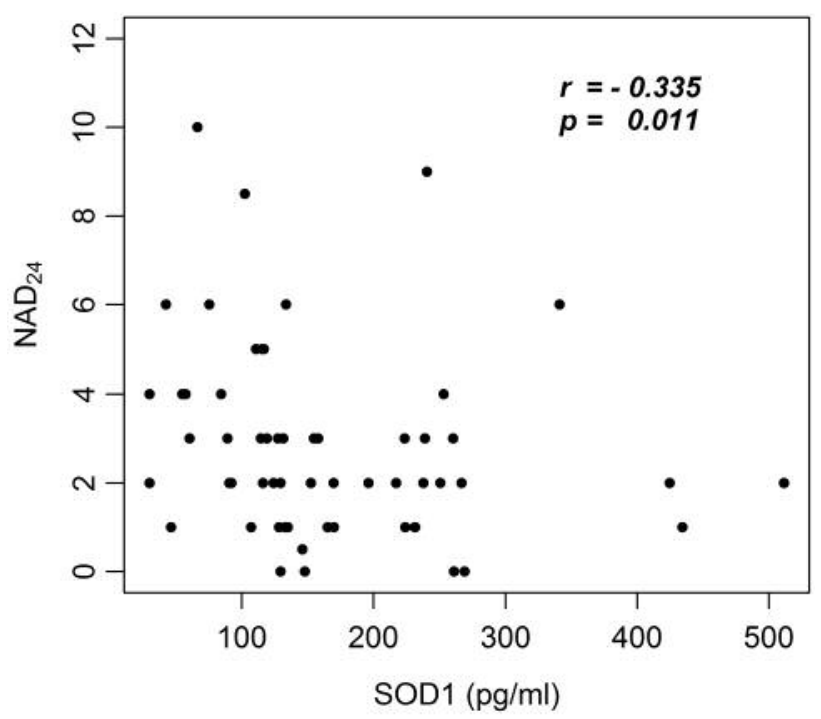

Figure 3. Scatter plots of the post-operative (POP2) plasma superoxide dismutase (SOD1) values versus number of analgesic doses during the first $24 \mathrm{~h}$ postoperatively $\left(\mathrm{NAD}_{24}\right)$ in cholecystectomy patients $(r=-0.335, p=0.011)$.

Table III. The postoperative alteration of plasma hs-CRP and SOD1 marker levels in the groups combined (all patients). Plasma levels were measured before operation (PRE), immediately after operation (POP1) and 6 h after operation (POP2). Median (interquartile range) values are shown. The Wilcoxon signed rank test was used.

\begin{tabular}{lccr}
\hline Marker & All patients & Alteration & $p$-Value \\
\hline Hs-CRP (mg/l) & & & \\
PRE & $1.35(0.49-3.15)$ & PRE vs. POP1 & 0.021 \\
POP1 & $1.45(0.50-3.03)$ & POP1 vs. POP2 & $<0.001$ \\
POP2 & $2.35(0.98-4.65)$ & & \\
SOD1(pg/ml) & & & \\
PRE & $149.97(97.2-209.6)$ & PRE vs. POP1 & 0.027 \\
POP1 & $171.2(114.7-256.7)$ & POP1 vs. POP2 & $<0.001$ \\
POP2 & $134.5(97.9-216.5)$ & & \\
& & & \\
& & &
\end{tabular}

LC is the gold standard for the treatment of symptomatic gallstone disease, although MC has been shown to have a similar early recovery after surgery (26-35). Several markers and methods have been used in the early detection of systemic oxidative stress. Polat et al. (10) assessed the effect of different increased intra-abdominal pressure (IAP) on lipid peroxidation and protein oxidation status during laparoscopic cholecystectomy. 24 patients who underwent LC with either 10 or $15 \mathrm{mmHg}$ of IAP were randomized into two groups. No difference was found between groups on lipid peroxidation or protein oxidation status. 
Koksal et al. (11) compared the effects of sevoflurane and desflurane on lipid peroxidation and protein oxidation status during laparoscopic cholecystectomy. Patients were randomly allocated to be in sevoflurane $(n=20)$ or desflurane $(n=20)$ study groups. No significant differences were found in SOD1 in the samples from bronchoalveolar lavage. Yagmurdur et al. (12) studied the effect of etomidate (group $1, \mathrm{n}=12$ ), thiopentoal (group 2, $\mathrm{n}=12$ ) and propofol (group 3, $\mathrm{n}=12$ ) on hypoperfusion-reperfusion phenomenon in the LC patients. There was significant increase in plasma malondialdehyde (MDA) levels in group 1 and MDA plasma levels were significantly decreased in group 3, suggesting that propofol could offer many advantages inhibiting lipid peroxidation and ROS formation.

Bickel et al. (13) assessed the reduction of the oxidative stress by intermittent pneumatic compression device (ISPC) in LC patients. IAP during LC may lead to decreased cardiac output and visceral perfusion and possible hypoperfusionreperfusion effect. $20 \mathrm{LC}$ patients were randomized into 2 groups; control group without ISPC $(n=10)$ and study group with ISPC $(n=10)$. There was no significant difference between study groups in liver enzymes, bilirubin or in hemodynamic patterns. In the control group increased lipid peroxidation level were noted post-operatively in comparison to preoperative levels, while in the study group such changes were not shown $(p=0.002)$.

Earlier, we reported the pain at rest and the $\mathrm{NAD}_{24}$ in the LC versus MC patients and our results suggest relatively similar results between these two groups (19). Firstly, the aim of this study was to investigate the plasma SOD1 levels in $\mathrm{LC}$ and MC patients versus cancer patients and secondly, to determine a correlation between the plasma SOD1 levels and analgesic doses during the first $24 \mathrm{~h}$ postoperatively $\left(\mathrm{NAD}_{24}\right)$ in gallstone patients undergoing cholecystectomy. The SOD1 levels increased by $14.1 \%$ immediately after surgery and then decreased by $21.3 \%$ at $6 \mathrm{~h}$ postoperatively. However, the plasma SOD1 levels preoperatively and following surgery in the LC versus MC patients were quite similar supporting the quite similar oxidative stress concept between these two cholecystectomy techniques. Interestingly, a highly significant inverse correlation between the individual $\mathrm{NAD}_{24}$ values and plasma SOD1 values postoperatively in operated patients was found. This could suggest that enhanced oxidative balance following surgery may have a protective role against postoperative pain.

The significant enhancement in the SOD levels, but not in the GPX levels after cholecystectomy is a new finding, although the rise in the SOD levels following surgery is in line with the results of Turker et al. (36) in patients undergoing open heart surgery with cardiopulmonary bypass. Woźniak et al. (37) found the SOD plasma levels to alter one week postoperatively, while the GPX levels stayed markedly lower than before surgery.
In conclusion, the cholecystectomy does significantly alter the oxidative stress marker SOD1 plasma levels immediately after operation, but the levels drop back $6 \mathrm{~h}$ following surgery. The plasma SOD1 levels preoperatively and following surgery in the LC and MC patients versus cancer patients were quite similar. Interestingly, cholecystectomy patients with higher levels of SOD1 appeared to have lower number of analgesic oxycone doses during the first $24 \mathrm{~h}$ postoperatively $\left(\mathrm{NAD}_{24}\right)$, which could suggest that enhanced oxidative balance following surgery may have a protective role against postoperative pain.

\section{Conflicts of Interest}

The Authors report no conflicts of interest or financial ties to disclose. The Authors alone are responsible for the content and writing of this article.

\section{Acknowledgements}

The study was funded by the Heikki, Aino and Aarne Korhonen foundation and the EVO-funding of the Helsinki and Kuopio University Hospital.

\section{References}

1 Stinton LM and Shaffer EA: Epidemiology of gallbladder disease: cholelithiasis and cancer. Gut Liver 6: 172-187, 2012.

2 Ros A and Nilsson E: Abdominal pain and patient overall and cosmetic satisfaction one year after cholecystectomy: outcome of a randomized trial comparing laparoscopic and minilaparotomy cholecystectomy. Scand J Gastroenterol 39: 773-777, 2004.

3 Harju J, Juvonen P, Kokki H, Remes V, Scheinin T and Eskelinen M: Minilaparotomy cholecystectomy with ultrasonic dissection versus conventional laparoscopic cholecystectomy: a randomized multicenter study. Scand J Gastroenterol 48: 1317-1323, 2013.

4 Rosenmüller MH, Thorén Örnberg M, Myrnäs T, Lundberg O, Nilsson E and Haapamäki MM: Expertise-based randomized clinical trial of laparoscopic versus small-incision open cholecystectomy. Br J Surg 100: 886-894, 2013.

5 Grune T: Oxidants and antioxidative defense. Hum Exp Toxicol 21: 61-62, 2002.

6 Valko M, Leibfritz D, Moncol J, Cronin MTD, Mazur M and Telser $\mathrm{J}$ : Free radicals and antioxidants in normal physiological functions and human disease. Int J Biochem Cell Biol 39: 44-84, 2007.

7 Robertson RP and Harmon JS: Diabetes, glucose toxicity, and oxidative stress: A case of double jeopardy for the pancreatic islet beta cell. Free Radic Biol Med 41: 177-184, 2006.

8 Leverve X: Hyperglycemia and oxidative stress: complex relationships with attractive prospects. Intensive Care Med 29: 511-514, 2003.

9 Yiannakopoulou EC, Nikiteas N, Perrea D and Tsigris C: Effect of laparoscopic surgery on oxidative stress response: systematic review. Surg Laparosc Endosc Percutan Tech 23: 101-108, 2013.

10 Polat C, Yilmaz S, Serteser M, Koken T, Kahraman A and Dilek ON: The effect of different intraabdominal pressures on lipid peroxidation and protein oxidation status during laparoscopic cholecystectomy. Surg Endosc 17: 1719-1722, 2003. 
11 Koksal GM, Sayilgan C, Aydin S, Uzun H and Oz H: The effects of sevoflurane and desflurane on lipid peroxidation during laparoscopic cholecystectomy. Eur J Anaesthesiol 21: 217-220, 2004.

12 Yagmurdur H, Cakan T, Bayrak A, Arslan M, Baltaci B, Inan N and Kilinc K: The effects of etomidate, thiopental, and propofol in induction on hypoperfusion-reperfusion phenomenon during laparoscopic cholecystectomy. Acta Anaesthesiol Scand 48: 772777, 2004.

13 Bickel A, Drobot A, Aviram M and Eitan A: Validation and reduction of the oxidative stress following laparoscopic operations: a prospective randomized controlled study. Ann Surg 246: 31-35, 2007.

14 Aspinen S, Harju J, Juvonen P, Selander T, Kokki H, Pulkki K and Eskelinen MJ: The plasma 8-OHdG levels and oxidative stress following cholecystectomy: a randomised multicentre study of patients with minilaparotomy cholecystectomy versus laparoscopic cholecystectomy. Scand J Gastroenterol 51: 1507-1511, 2016.

15 Purdy M, Kokki M, Anttila M, Aspinen S, Juvonen P, Selander T, Kokki H, Pulkki K and Eskelinen M: Does post-surgery placement of rectus sheath block analgesia alter the oxidative stress biomarker 8-OHdG concentrations: A randomised trial of patients with cancer and benign disease. Cancer Genomics Proteomics 13: 239-244, 2016.

16 Purdy M, Kärkkäinen J, Kokki M, Anttila M, Aspinen S, Juvonen P, Kokki H, Pulkki K, Rantanen T and Eskelinen M: Does rectus sheath block analgesia alter levels of the oxidative stress biomarker glutathione peroxidase: A randomised trial of patients with cancer and benign disease. Anticancer Res 37: 897902, 2017.

17 Kärkkäinen J, Aspinen S, Harju J, Juvonen P, Pulkki K and Eskelinen M: Plasma glutathione peroxidase (GPX1) levels and oxidative stress in gallstone patients operated with two different cholecystectomy techniques: A Randomized study with special reference to cancer patients. Anticancer Res 37: 6921-6927, 2017.

18 Kärkkäinen J, Selander T, Purdy M, Juvonen P and Eskelinen M: Patients with increased levels of the oxidative stress biomarker SOD1 appear to have dimished postoperative pain after midline laparotomy: A randomized trial with special reference to postoperative pain score (NRS). Anticancer Res 38: 1003-1008, 2018.

19 Aspinen S, Harju J, Kinnunen M, Juvonen P, Kokki H and Eskelinen M: A randomized multicenter study of minilaparotomy cholecystectomy versus laparoscopic cholecystectomy with ultrasonic dissection in both groups. Scand J Gastroenterol 51: 354-359, 2016.

20 Collins A: Comparison of different methods of measuring 8oxoguanine as a marker of oxidative DNA damage. ESCODD (European Standards Committee on Oxidative DNA Damage). Free Radic Res 32: 333-341, 2000.

21 Fehér J, Lengyel G and Blázovics A: Oxidative stress in the liver and biliary tract diseases. Scand J Gastroenterol Suppl 228: 3846, 1998.

22 Mamarelis I, Pissaridi K, Dritsa V, Kotileas P, Tsiligiris V, Tzilalis $\mathrm{V}$, and Anastassopoulou J: Oxidative stress and Atherogenesis. An FT-IR Spectroscopic Study. In Vivo 24: 883-888, 2010.

23 Sesti F, Tsitsilonis OE, Kotsinas A, and Trougahos I: Review: Oxidative Stress-mediated Biomolecular Damage and Inflammation in Tumorigenesis. In Vivo 26: 395-402, 2012.

24 Pace MC, Passavanti MB, Aurilio C, Sansone P, Aurilio R, De Maria S, Lama S, Federico A, Ravagnan GP, Caraglia M, and Stiuso P: Polydatin administration improves serum biochemical parameters and oxidative stress markers during chronic alcoholism: A pilot study. In Vivo 29: 405-408, 2015.

25 Berger MM and Shenkin A: Update on clinical micronutrient supplementation studies in the critically ill. Curr Opin Clin Nutr Metab Care 9: 711-716, 2006.

26 Vagenas K, Spyrakopoulos P, Karanikolas M, Sakelaropoulos G, Maroulis I and Karavias D: Mini-laparotomy cholecystectomy versus laparoscopic cholecystectomy: which way to go? Surg Laparosc Endosc Percutan Tech 16: 321-324, 2006.

27 Harju J, Pääkkönen M and Eskelinen M: Minilaparotomy cholecystectomy as a day surgery procedure: a prospective clinical pilot study. Scand J Surg 96: 206-208, 2007.

28 Harju J, Pääkkönen $M$ and Eskelinen M: Comparison of the quality of life after minilaparotomy cholecystectomy versus laparoscopic cholecystectomy: a prospective randomized study. Isr Med Assoc J 9: 147-148, 2007.

29 Harju J, Kokki H, Pääkkönen M, Karjalainen K and Eskelinen M: Feasibility of minilaparotomy versus laparoscopic cholecystectomy for day surgery: a prospective randomised study. Scand J Surg 99: 132-136, 2010.

30 Harju J, Juvonen P, Kokki H, Remes V, Scheinin T and Eskelinen M: Minilaparotomy cholecystectomy with ultrasonic dissection versus conventional laparoscopic cholecystectomy: a randomized multicenter study. Scand J Gastroenterol 48: 1317-1323, 2013.

31 Harju J, Aspinen S, Juvonen P, Kokki H and Eskelinen M: Tenyear outcome after minilaparotomy versus laparoscopic cholecystectomy: a prospective randomised trial. Surg Endosc 27: 2512-2516, 2013.

32 Aspinen S, Harju J, Juvonen P, Karjalainen K, Kokki H, Paajanen $\mathrm{H}$ and Eskelinen $\mathrm{M}$ : A prospective, randomized study comparing minilaparotomy and laparoscopic cholecystectomy as a day-surgery procedure: 5-year outcome. Surg Endosc 28: 827-832, 2014.

33 Aspinen S, Harju J, Juvonen P, Kokki H, Remes V, Scheinin T and Eskelinen M: A prospective, randomized multicenter study comparing conventional laparoscopic cholecystectomy versus minilaparotomy cholecystectomy with ultrasonic dissection as day surgery procedure - 1-year outcome. Scand J Gastroenterol 49: 1336-1342, 2014.

34 Aspinen S, Kinnunen M, Harju J, Juvonen P, Selander T, Holopainen A, Kokki H, Pulkki K and Eskelinen M: Inflammatory response to surgical trauma in patients with minilaparotomy cholecystectomy versus laparoscopic cholecystectomy: a randomised multicentre study. Scand J Gastroenterol 51: 739-744, 2016.

35 Aspinen S, Kärkkäinen J, Harju J, Juvonen P, Kokki H and Eskelinen M: Improvement in the quality of life following cholecystectomy: a randomized multicenter study of health status (RAND-36) in patients with laparoscopic cholecystectomy versus minilaparotomy cholecystectomy. Qual Life Res 26: 665-671, 2017.

36 Türker FS, Doğan A, Ozan G, Kıbar K and Erışır M: Change in Free Radical and Antioxidant Enzyme Levels in the Patients Undergoing Open Heart Surgery with Cardiopulmonary Bypass. Oxid Med Cell Longev 2016: 1783728, 2016.

37 Woźniak B, Woźniak A, Mila-Kierzenkowska C and Kasprzak HA: Correlation of oxidative and antioxidative processes in the blood of patients with cervical spinal cord injury. Oxid Med Cell Longev 2016: 6094631, 2016.

Received April 6, 2018

Revised May 7, 2018

Accepted May 8, 2018 